\title{
Neural Network Based Lna Design for Mobile Satellite Receiver
}

\author{
Abhijeet Upadhya \\ M.Tech. Scholar, E.C.E \\ Ajay Kumar Garg Engineering College, \\ Ghaziabad, India
}

\author{
Prof. P. K. Chopra \\ Head of Department, E.C.E. \\ Ajay Kumar Garg Engineering College, \\ Ghaziabad, India
}

\begin{abstract}
Paper presents a Neural Network Modelling approach to microwave LNA design. To acknowledge the specifications of the amplifier, Mobile Satellite Systems are analyzed. Scattering parameters of the LNA in the frequency range 0.5 to $18 \mathrm{GHz}$ are calculated using a Multilayer Perceptron Artificial Neural Network model and corresponding smith charts and polar charts are plotted as output to the model. From these plots, the microwave scattering parameter description of the LNA are obtained. Model is efficiently trained using Agilent ATF 331M4 InGaAs/InP Low Noise pHEMT amplifier datasheet and the neural model's output seem to follow the various device characteristic curves with high regression. Next, Maximum Allowable Gain and Noise figure of the device are modelled and plotted for the same frequency range. Finally, the optimized model is utilized as an interpolator and the resolution of the amplifying capability with noise characteristics are obtained for the L Band of MSS operation.
\end{abstract}

Keywords-Satellite; Mobile; Artificial Neural Networks; Scattering Parameters; Noise Figure

\section{INTRODUCTION}

Historically, Mobile Satellite Systems (MSSs) have been used in Marine and Military Communications for providing the seamless connectivity to mobile users in regions where no other means of network can be established. MSS have also been successfully utilized in disaster management situations when all other forms of communication systems fail to respond. Recently, Hybrid Mobile Satellite Systems (HMSS) [1], [2], [3] have been opted in several countries due to the possibility of retrieving the combined advantages of terrestrial Cellular networks and Satellite Systems. Cellular Systems provide high bandwidth, low latency data and voice services. On the other hand, use of Satellite Systems extends the geographical coverage of the overall network. MSS systems like Thuraya, ACeS, and INMARSAT etc. have contributed a lot in the development of hybrid network by coordinating with cellular vendors across the globe.

Choosing the right equipment with right service is the key to growth in mobile industry. Dual Mode Sat Phones combine the functional benefits of the cellular-based equipment with the advantage of reliable and uniform coverage provided by extended foot print of the Satellite. Mobile Terminal must facilitate a seamless handover between the two types of communication systems as and when required.

The main aim of the present work is to obtain insights about Satellite links and their availability issues. Henceforth, a
Low Noise Amplifier's model is created and optimized using Artificial Neural Networks (ANN) based learning in the frequency range starting from $500 \mathrm{MHz}$ to $18 \mathrm{GHz}$. The advantages of Neural Networks (NN) when compared to conventional methods lies in the ease with which they may be modelled to generalize any nonlinear relationship between variables. NN models have existed over six decades after McCulloch and Pitts [4] presented the model of the basic human nervous system and its basic unit termed a neuron. But implementation of models based on ANN approach in design of a microwave devices have been reported only in the last decade and a half [5], [6], [7].

Modelling issues encountered using other methods such as excessive time consumption, required level of designer's expertise, etc. disappear due to Universal Approximation capability of the of the NN [8]. One of the best works ever presented in literature in [9], the authors have demonstrated a step by step method of how NN may be obtained for understanding the behavior of a Radio Frequency (RF) device. This research work we have used Agilent ATF 331M4 pHEMT made of InGaAs/InP material.

The remaining of the paper is segmented in the following manner. Section II carries a review of the various degradations that the Satellite signal suffers. Section III and IV provide RF receiver and LNA design issues while section $\mathrm{V}$ discusses ANN modelling in brief. Finally, simulated results are presented in section VI and VII.

\section{SATELLITE LINK IMPAIRMENTS}

To incorporate a low cost, high spectrally efficient wireless communication system, a complete description of all the propagation impairments is necessary. Satellite signal consists of a direct component, called Line of Sight (LoS), and multiple copies of this direct component known as multipath components. In [10], the authors have modelled the received Satellite signal as

$C(t)=A_{0} e^{j\left(\omega_{0} t+\varphi_{0}\right)}+\sum_{m=1}^{M} A_{m} e^{j\left(\omega_{m} t+\varphi_{m}\right)}+n(t)$

Where $A_{0}, \omega_{0}, \varphi_{0}$ are amplitude, Doppler shift and phase of the LoS component, while summation part quantifies the ' $M$ ' multipath components and $n(t)$ is the White Gaussian Noise.

According to equation (1), the signal received by the mobile handset receiver comprises of a dominating LoS and several other components shifted in frequency and relative phase. Following are some link degrading factors: 


\section{A. Free Space Path Loss}

FSL (Free Space Loss) is determined by the amount of distance that the signal has to travel and on the system's operating frequency. High operating frequency and larger distance results in higher losses. Even in the LoS component of received signal, FSL impacts the signal strength and mobile receiver front end must provide the legitimate amplification in order to perceive the information.

\section{B. Ionospheric Effects}

The ionized section of the atmosphere extending from a height of $30 \mathrm{~km}$ to $1000 \mathrm{~km}$ has adverse effects on earthSatellite radio propagation. Two of the more important signal effecting mechanisms encountered by the signal propagating in ionosphere are Scintillation and Polarization depending upon impact of individual layer over the signal. Due to high inhomogeneity of Ionosphere, signal varies in amplitude, phase and angle of arrival as it propagates causing what is called Ionospheric Scintillations, the effects of which decreases with increase in frequency [11]. Rotation of plane of polarization of the EM (Electro-magnetic) wave as it passes through the irregular motion of free electrons in Ionosphere under the influence of earth's magnetic field degrades the spectral efficiency of the Satellite radio channels.

\section{Tropospheric Meteorites}

Lower portion of earth's atmosphere extending upto a height of $15 \mathrm{~km}$ above sea level introduces many hydrometeoric effects such as those of clouds, rain, snow, fog etc. to the propagating radio signal and becomes significant above frequency of $1 \mathrm{GHz}$. Ippolito [11] suggests many models for quantifying atmospheric attenuation. At operating frequency above $10 \mathrm{GHz}$ (e.g. Ku Band), rain attenuation effects Satellite communication links adversely as the radio wave energy is absorbed and scattered by rain drops.

\section{Sun Outage}

Radiations from the Sun effects the Satellite signal which ranges from partial degradation to total destruction. The nonionizing component of the radiation consists of electromagnetic waves providing a constant source of heating to the part of the spacecraft facing the Sun with a rate of 1400 W. With respect to MSS systems, Sun Transits/ Outages/ Fades are of more concern as they interfere with the Geo Stationary Satellite Signals and in some cases during the equinoxes tend to completely interrupt them.

\section{E. Multipath Fading}

Received radio signal in urban dense environment is the resultant of diffused scattered waves produced from LoS component. Combining constructively or destructively, these multipath components lead to certain signal level at mobile receiver front end and must be considered during decision over RF component's specifications is carried.

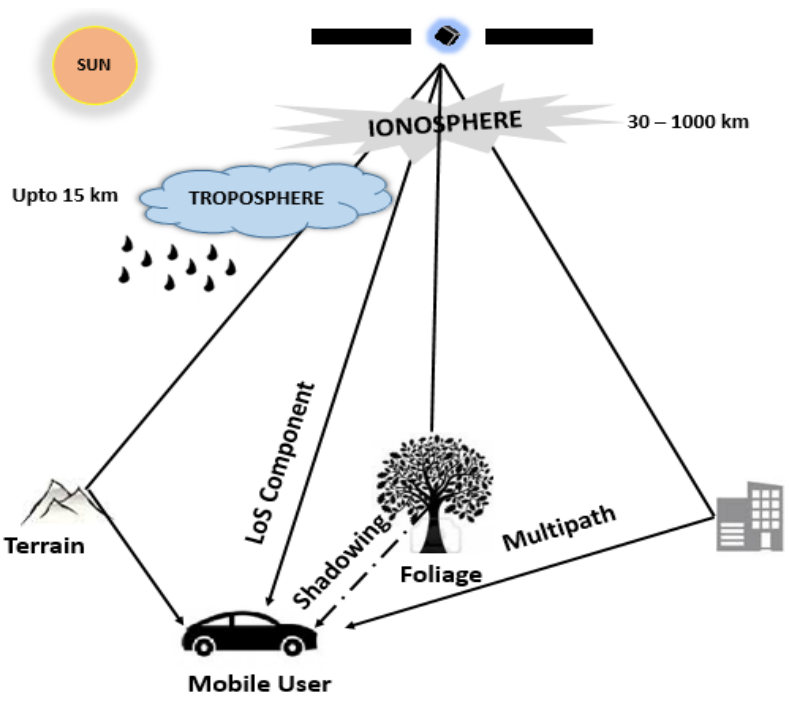

Fig. 1. Mobile Satellite Scenario

\section{F. Shadowing}

MSS face complete demolition of the LoS radio signal strength due to presence of large obstacles in the LoS between the Satellite and the mobile user. Vegetation and foliage cause extensive signal absorption. Many mathematical models have been demonstrated in literature to quantify the effect of shadowing [12]. Factors discussed in this segment are illustrated in Fig. 1.

\section{MOBILE RECEIVER FRONT END}

MSS reception at the mobile terminal is affected by several key factors as discussed in the previous section. Schemes must be opted to overcome the same at the mobile receiver handset. In order to integrate the best of the functionalities of the Satellite and Cellular receiver, generally the architecture shown in Fig. 2 is opted. In this cost effective architecture, the user level selection of the preferred network is possible with Common Control unit in the uplink. While on the downlink, generally this issue is controlled by the network level management entities. Some carriers also implement dual LNA in the separate Satellite links but authors believe that this is not an economically viable solution.

Terminals supporting Dual Mode are designed to cater seamless handover between Cellular and Satellite networks. Two types of implementations are noted i.e. a Hybrid solution and an Integrated solution. Hybrid implementation is more complex than the later due to the fact that although the cellular-satellite switching intelligence is housed in a single enclosure, they work as stand-alone terminals. Integrated solution has recently gained momentum because of their simplicity due to integration of cellular and satellite modems over a single wafer resulting in better inter-network-switching characteristics with reduced cost. 


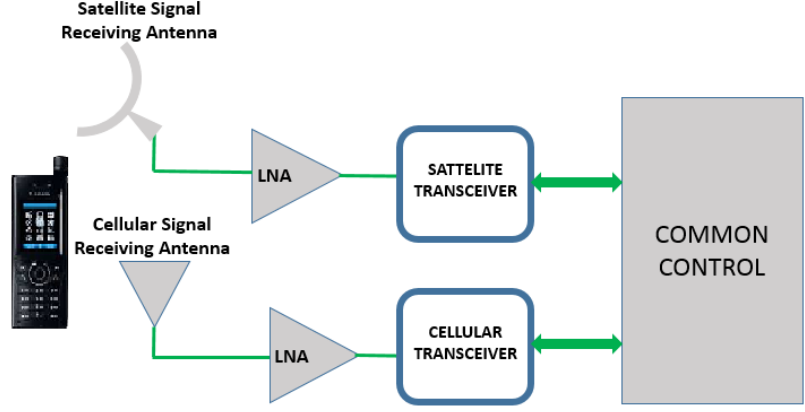

Fig. 2. Mobile RF Front End

\section{LNA DESIGN}

In order to relax the Satellite link margin, the LNA must assist with a minimum amount of gain and keep the noise figure below a predefined level. The characterization of the microwave device is generally performed using the Scattering (S) parameters as obtaining the short circuit or an open circuit for devices operating at microwave frequencies is not practically feasible. For a two port network, four complex $\mathrm{S}$ parameters are defined and when analysed, provide correct insight to device performance. $S_{11}$ and $S_{22}$ provide insights about the reflection from the device while $S_{12}$ and $S_{21}$ are reverse and forward transmission gains respectively. Reflection coefficient quantifies the mismatch at concerned port and is represented on Smith chart. Remaining two $\left(S_{12}\right.$ and $S_{21}$ ) are plotted on Polar plots for simplicity. Next, some important aspects of LNA design are considered and described in brief.

\section{A. Key Parameters}

Input to the LNA is the high frequency noise effected received signal at the load end of the RF antenna. According to the famous Friis Formula, the noise performance of an RF receiver is determined by the Noise Figure (NF) and Gain of the LNA. Minimization of NF and maximization of the Maximum Available Gain (MAG) generally have opposite requirements as discussed in [13]. Minimum NF is obtained when the LNA input impedance is made equal to the optimum impedance $Z_{\text {opt }}$ calculated at operating frequency. On contrary to this, MAG is obtained under perfectly matched input and output terminations in characteristic impedance $Z_{0}$. These two complex impedances are never equal and an optimization scheme needs to be addressed.

\section{B. Selection of Semiconductor Material}

With the evolution of nanometre technology, the Monolithic Microwave Integrated Circuits (MMIC), a scaled down solution to integration several RF components on silicon substrate is possible. Microwave amplifier device technology brings the High Electron Mobility Transistor (HEMT) to achieve very fast switching speeds using compound semiconductors for crystal growth. Gallium Arsenide (GaAs) was the first to be opted for its high carrier mobility and remains to lead the lot. Recently, wide band semiconductor materials such as Silicon Carbide $(\mathrm{SiC})$ and Gallium Nitride $(\mathrm{GaN})$ have emerged as robust options at high power withstanding capabilities. Present research work has opted InGaAs based material which is an alloy of GaAs and Indium
Arsenide (InAs) which has superior performance for space applications. InAs/GaAs alloy is described as $\operatorname{In}_{\mathrm{x}} \mathrm{Ga}_{1-\mathrm{x}} \mathrm{As}$ where ' $\mathrm{x}$ ' is the proportion of InAs and ' $1-\mathrm{x}$ ' is the proportion of GaAs. Literature survey confirms the fact that most convenient substrate for InGaAs is InP (Indium Phosphide) and the combination is termed InGaAs/InP. Due to its advantages in terms of low noise and high gain, InGaAs/InP has recently gained recognition in space applications [14].

\section{Design Methodology}

As discussed in segment A of the present section, the different parameters important for designing the LNA are of opposing in nature. Different optimization schemes are demonstrated in [15], where the authors advocated Power Constrained optimization scheme for the design of active amplifier. Since the Voltage Standing Wave Ratio (VSWR) quantifies the amount of mismatch of the device ports taking the characteristic impedance as reference value, in [16], a practical trade-off between NF and VSWR is claimed using a graphical approach. Designer must also take into consideration various other aspects like bias conditions, device geometry, operating bandwidth, RF power generated etc. all at a time Due to the complexity involved, generally an unacceptable amount of round off error results during the optimization process. Also, the models are restricted to specific case study and may not be generalized. Keeping the above stated under consideration, Artificial Neural Network presents an accurate, flexible approach to design active device models. The Universal Approximation property given in [8] forms the basis of employing a Neural Network (NN) for a generalized model. Next section investigates ANN models.

\section{ANN LNA MODEL}

Basic processing unit of the human nervous system is a biological neuron. In 1942, the first man made neuron model was presented [4] and till date the human investigation to imitate the complex parallel processing nature of the human nervous system remains a topic for research.

In order to generalize a massive interconnection between input and output variables, a Back Propagation algorithm [9] is utilized. An interconnection of various layers of artificial neurons is formed with the first layer accepting the input variables and is termed the Input layer while the layer at which output parameters are obtained is termed the Output layer. All the layers existing between these layers are called the Hidden layers and optimization of the model depends upon the number of Hidden layers and the value of the weights and biases of individual neurons in this layer. The ANN model must be trained with accurately measured values of desired output variables corresponding to input parameters. When optimized, the model has the capability to generalize the input/output nonlinear relationship [17], [18].

An innovative approach to an LNA design was presented in [19], where the model based on input variables frequency and temperature of device operation and biasing voltage and current was implemented. Training of this model performed in present paper utilizes this concept to optimize the model in order to retrieve Scattering parameters, NF and MAG of the LNA. 
Fig 3 shows a schematic representation of modelling strategy opted in present work. The NN training is based on experimental data as a function of frequency of operation, operating temperature, drain to source voltage and drain current of the pHEMT amplifier. Parameters are varied between some predefined ranges. It is only due to such modelling that a neural model helps in simultaneous optimization of several variables involved.

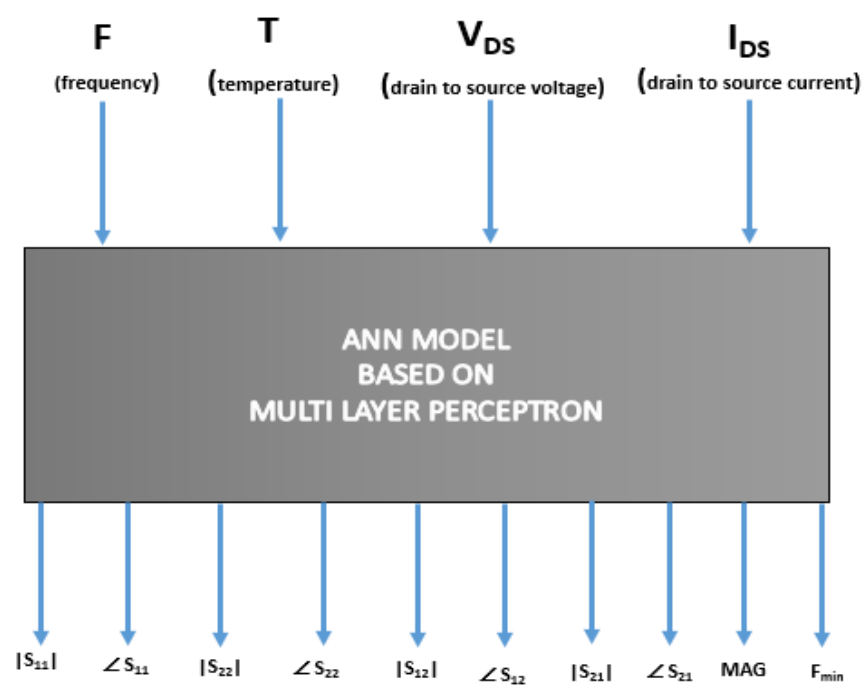

Fig. 3. ANN Modelling Strategy

\section{RESULTS AND DisCUSSIONS}

In order to model the LNA, the training of the neural model is done using the Agilent ATF 331M4 pHEMT FET family datasheet values [20]. MATLAB ANN Tool Box is used as software platform for generating the feedforward network with Levenberg Marquardt learning algorithm. The mean square error (MSE) for the present model's learning gradient is set to $10^{-6}$ and network is optimized to plot the Smith charts and the Polar charts of the scattering parameters. Inputs to the model are frequency, biasing conditions and operating temperature.

Accuracy of the model increases with increase in training data set values and as may be easily followed from Fig. 4(a), 4(b), 4(c) and 4(d), the Neural Network is trained efficiently.

These models are optimized for biasing condition of 2 Volts $V_{D S}$ and $40 \mathrm{~mA} I_{D}$ for the pHEMT for the frequency range starting from $0.5 \mathrm{GHz}$ to $18 \mathrm{GHz}$. MAG and NF are also calculated and plotted in Figures 5(a) and 5(b) respectively for the same frequency range. The values of the microwave LNA MAG and NF, drop and rise with increase in operating frequency, respectively, which seems intuitively correct.

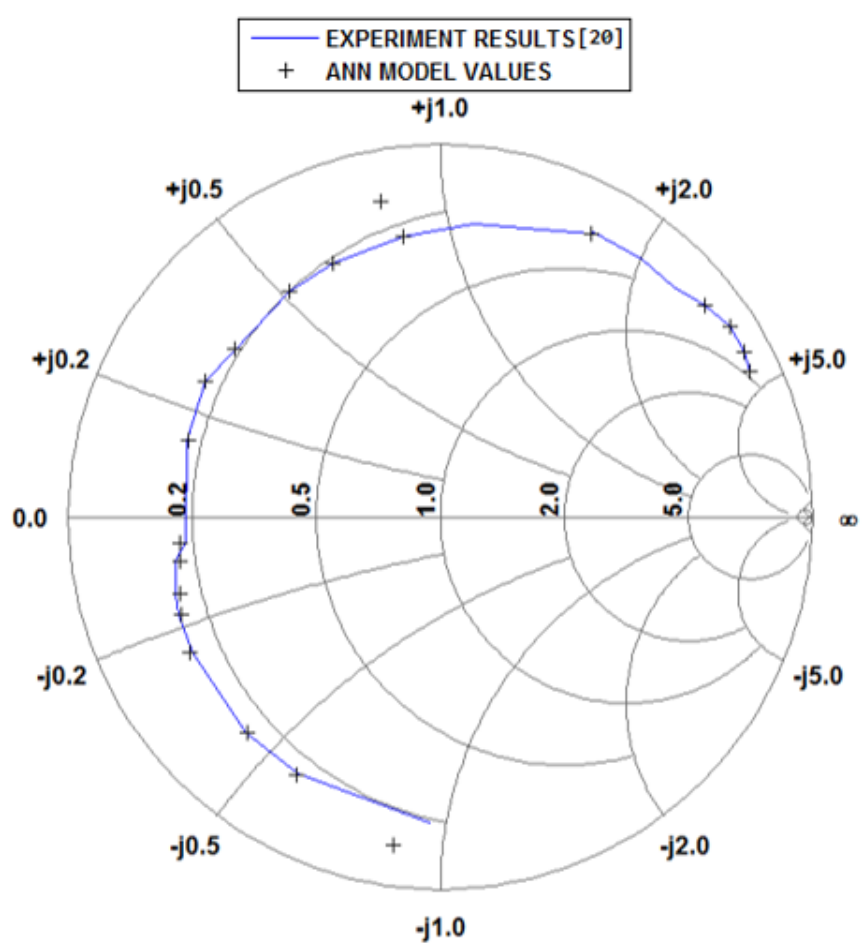

Fig. 4. (a). $\mathrm{S}_{11}$ Smith Chart

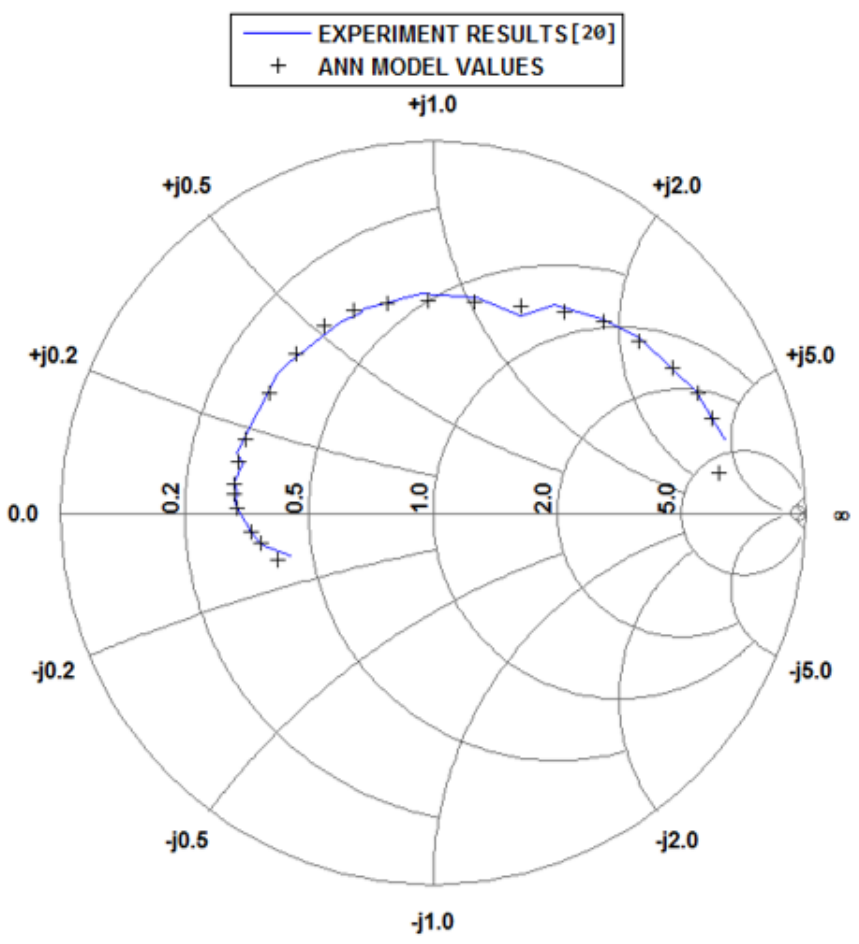

Fig. 4. (b) $\mathrm{S}_{22}$ Smith Chart 


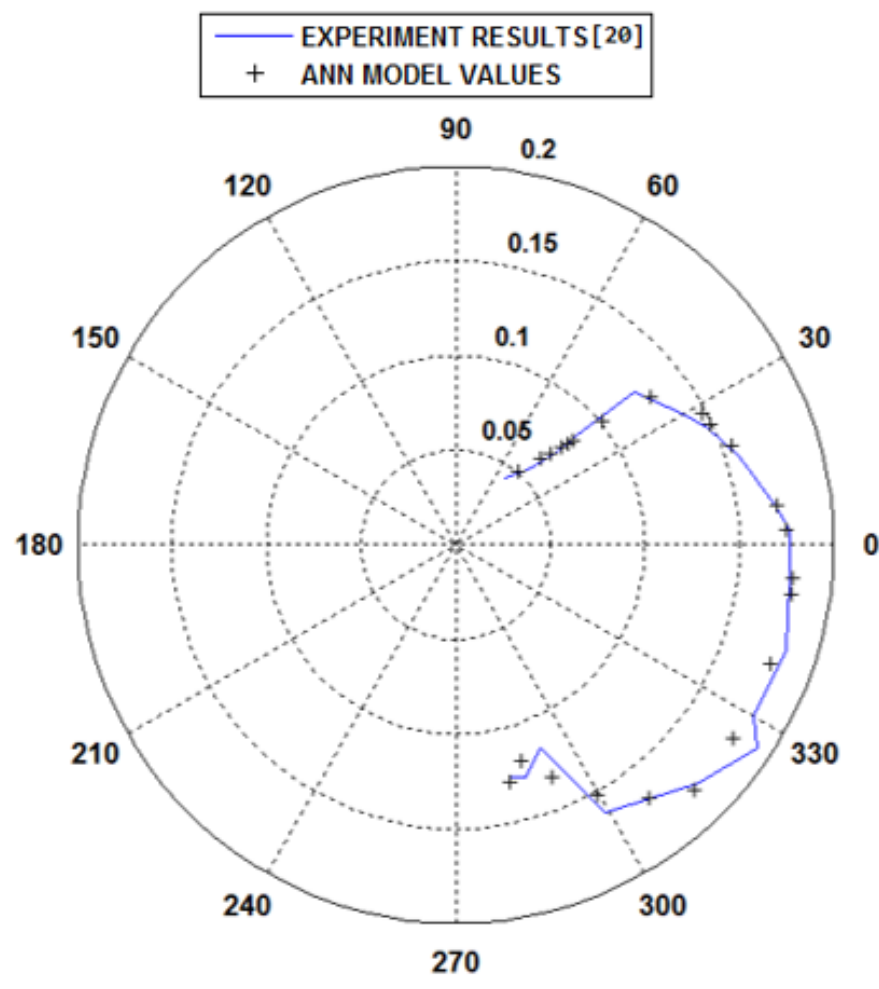

Fig. 4. (c). $\mathrm{S}_{12}$ Polar Plot

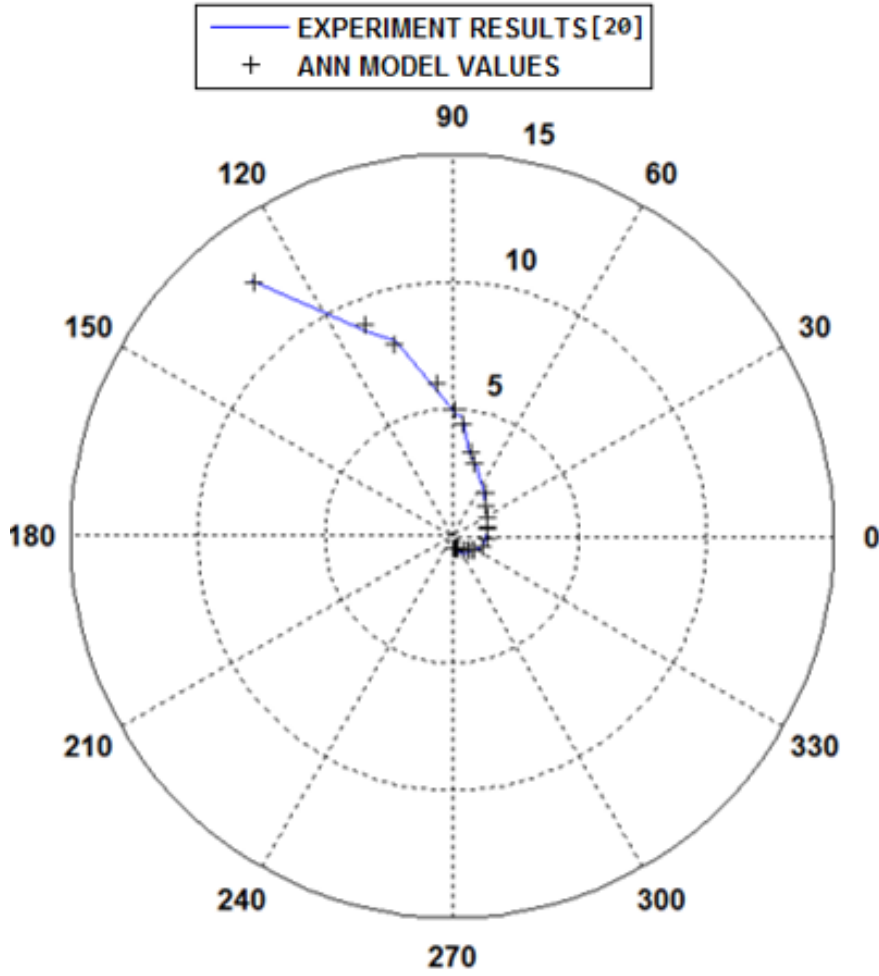

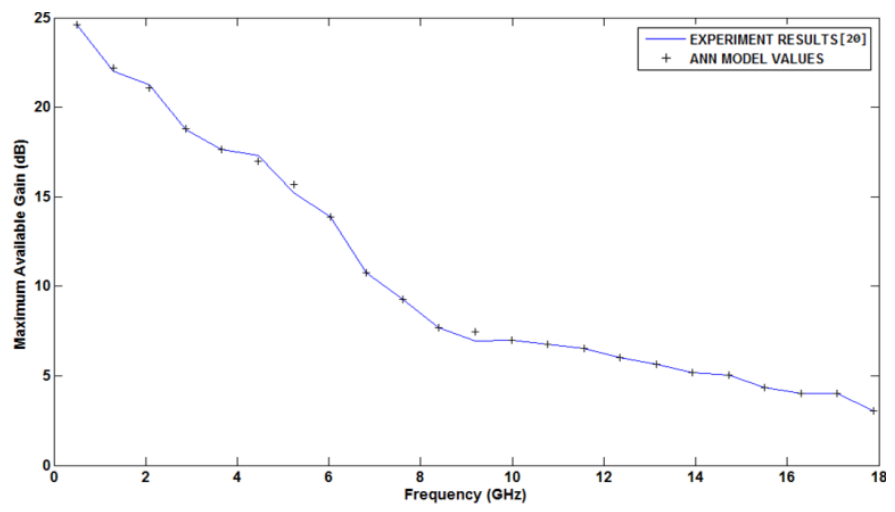

Fig. 5. (a). MAG (dB) vs Frequency $(\mathrm{GHz})$

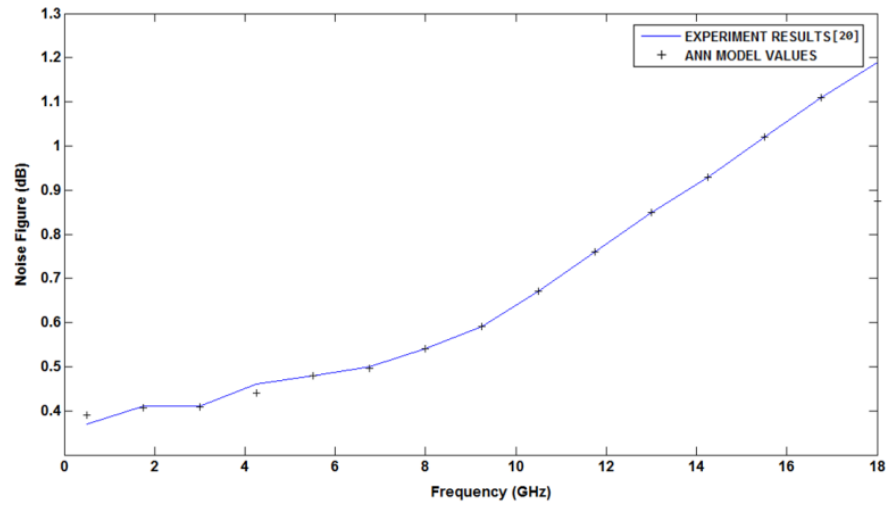

Fig. 5. (b). Noise Figure (dB) vs Frequency (GHz)

\section{PAPER CONTRIBUTION}

As discussed initially, the designed LNA is to be implemented in the RF front of the mobile receiver of a Dual Mode SatPhone. If the HMSS system is operated in the L Band designated by the ITU-R, then resolution of the results must be improved in the $2 \mathrm{GHz}$ spectrum. In order to achieve the same for the two most important parameters for LNA in MAG and NF, the model developed in section VI is used to interpolate the values in the concerned frequency range. The interpolated values are plotted in Fig. 6(a) and 6(b) for MAG and NF respectively with increased number of data set for 0 to $2 \mathrm{GHz}$ frequency range. By comparing Fig. 5(a) and 6(a) it may be clearly noticed that the latter is more informative than the former in the L Band when the LNA is to be analysed for amplification. Same is true for the resolution of NF in Fig. 5(b) and 6(b). The interpolation capabilities of the Neural Network may easily be noted. It is very important to note that before using the ANN model for interpolation, it was trained with all the datasheet values and the MSE (Mean Square Error), regression coefficients and error estimation on point to point basis is computed and optimized.

Fig. 4. (d). $S_{21}$ Polar Plot 


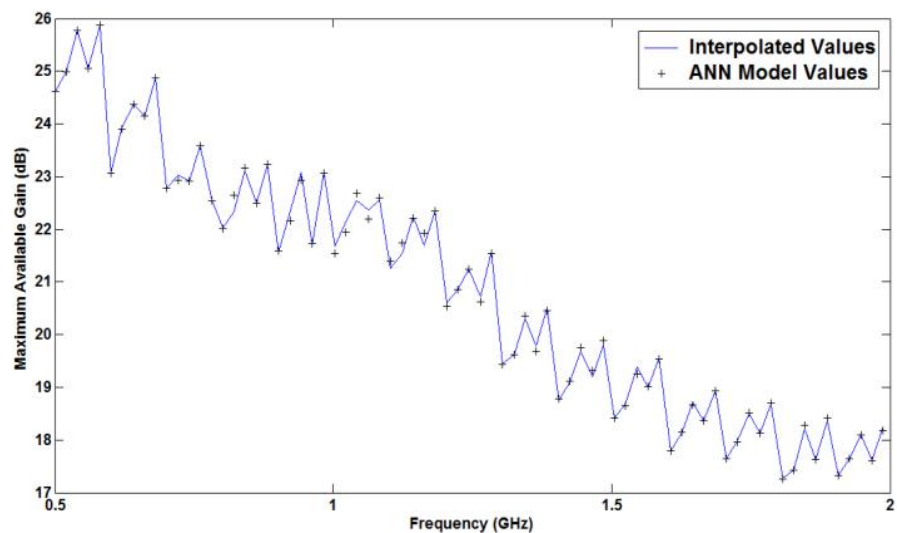

Fig. 6. (a). $\mathrm{MAG}(\mathrm{dB})$ vs Frequency $(\mathrm{GHz})$

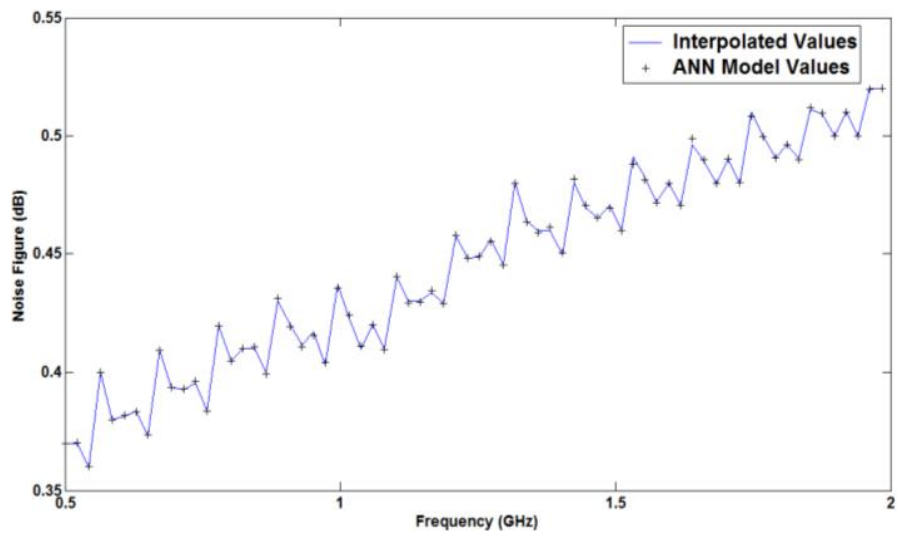

Fig. 6. (b). Noise Figure (dB) vs Frequency (GHz)

\section{CONCLUSION}

Signal deteriorating factors in Mobile Satellite System are discussed. A Hybrid MSS mobile receiver schemes are presented and an LNA is designed keeping in view its compatibility with the mobile unit's RF front end. A brief summary of Artificial Neural Network is provided to develop a quick insight into why designers are interested in such modelling strategy.

Optimization of the LNA model is carried out using the ANN approach. All the scattering parameters are calculated and their respective Smith charts and Polar plots are plotted and the results show that the ANN model has been trained efficiently. The trained model is utilized for interpolation to obtain performance analysis in the L Band range of MSS operation for Gain and Noise Figure of the pHEMT MMIC LNA. The performance of an LNA depends on the input and output matching networks, which is dictated by the scattering parameter description of the device. As a future scope to the present work, the authors recommend the design of matching networks for the LNA designed in the present work.

\section{ACKNOWLEDGMENT}

I sincerely thank Ajay Kumar Garg Engineering College, Ghaziabad for providing the opportunity and guidance for research work.

\section{REFERENCES}

[1] Sastri L. Kota, Giovanni Giambene, Paolo Chini, "A Mobile Satellite Systems Frame Work For Network CENTRIC Applications", Conference on Military Communications (MILCOM), IEEE, Volume., No., pp.1-8, Nov. 2008.

[2] Zhang Tao; Zhang Jun; Liu Zhong Kan, "A Delay Constraint Minimum Cost Routing Algorithm for Mobile Satellite Networks," Information, Communications and Signal Processing, IEEE 2005.

[3] Umehira, M.; Fujita, S.; Zhen Gao; Jing Wang, "Dynamic channel assignment based on interference measurement with threshold for multibeam mobile satellite networks," Asia-Pacific Conference on Communications (APCC), August, 2013.

[4] Warren S. McCulloch, Walter H. Pitts, "A Logical Calculus of the Ideas Immanent in Nervous Activity", Bulletin of Mathematical Biophysics, 1943.

[5] Zlatica Marinković, Aleksandar Stošić, Vera Marković, Olivera Pronić., "ANNs in Bias-Dependent Modeling of S-parameters of Microwave FETs and HBTs", Microwave Review, June 2006.

[6] Anil Ahlawat, Manoj Pandey, Sujata Pandey "A Fast and Robust Approach for Modeling of Nanoscale Compound Semiconductors for High Speed Digital Applications, Journal Of Semiconductor Technology And Science, 2006

[7] Ronan G. Brady, Christopher H. Oxley, Thomas J. Brazil 'An Improved Small-Signal Parameter-Extraction Algorithm for GaN HEMT Devices', IEEE Transactions on Microwave Theory and Techniques, Vol. 56, No. 7, July 2008.

[8] Kur Hornik, Maxwell Stinchcombe and Halber White, "Multilayer Feedforward Networks are Universal Approximators", Pergamon Press, 1989.

[9] Qi-Jun Zhang el. al., "Artificial Neural Networks for RF and Microwave Design-From Theory to Practice", IEEE Transaction on Microwave Theory and Techniques, 2003.

[10] Lu Lu, Daoxing Guo, Aijun Liu, Maoqiang Yang, "Analysis of Channel Model for GEO Satellite Mobile Communication System", National Conference on Information Technology and Computer Science, Atlantic Press, 2012.

[11] Dr. Louis J. Ippolito, "Propagation Effects Handbook For Satellite Systems Designs", ACTS Conference 2000, Sixth Ka-Band Utilization Conference, Cleveland, Ohio, Issue No. 2, 2008.

[12] Chun Loo. "A Statistical Model for a Land Mobile Satellite Link". Vehicular Technology, IEEE Transactions Volume. 34, Issue No. 3, 1985.

[13] Guillermo González,"Microwave Transistors Amplifiers: Analysis and Design", 2E, Prentice Hall, 1997

[14] Onur Esame, Yasar Gurbuz, Ibrahim Tekin, Ayhan Bozkurt, 'Performance Comparison of the state of art heterojunction bipolar devices (HBT) based on AlGaAs /GaAs, $\mathrm{Si} / \mathrm{SiGe}$ and InGaAs/InP', Microelectronics Journal, Elsevier, Nov., 2004.

[15] Trung-Kien Nguyen et. al., "CMOS Low - Noise Amplifier Design optimization Techniques" IEEE Transactions on Microwave Theory and Techniques, 2004.

[16] Alan Victor, "An Analytic and Graphical Method for LNA Design with Feedback" Summit Technical Media, High Frequency Electronics, 2010.

[17] Vikas Chaudhary, R.S. Bhatia, Anil Ahlawat, 'An efficient selforganizing map learning algorithm using the set of nearest neurons', International Conference on Contemporary Computing (IC3), Aug. 2013.

[18] Anil Ahlawat, Manoj Pandey, Sujata Pandey, 'AFast and Robust Approach for Modeling of Nanoscale Compound Semiconductors for High Speed Digital Applications', Journal of Semiconductor Technology and Science, IEEE, Sept., 2006.

[19] P. K. Chopra et. al. , "ANN Modeling Approach for Designing Low Noise pHEMT Amplifier in Wireless Communication Systems", Optical Memory and Neural Networks (Information Optics), Springer, 2011

[20] Agilent ATF 3311M4 Low Noise Pseudomorphic HEMT in a Miniature Leadless Package available at http://www.agilent.com. 